Ivana Vilić*

UDK : 811.113.1'367:811.163.41

Faculté de Philosophie et Lettres

Université de Novi Sad
DOI: $10.19090 /$ gff.2021.3.41-54

originalni naučni rad

\title{
LE MOUVEMENT DANS L'EXPRESSION DES RELATIONS SPATIALES EN FRANÇAIS ET EN SERBE
}

Cet article présente l'analyse de l'expression du mouvement en français et en serbe. Nous avons comparé l'expression de l'origine du déplacement, de son terme et de la trajectoire. L'analyse montre que dans les deux langues, à part le verbe, les prépositions et en serbe, le cas, jouent un rôle important dans l'expression de l'origine et du terme du déplacement. La plus grande différence s'observe dans l'expression de la trajectoire, qui est exprimée en français par le verbe et la préposition à, alors qu'en serbe elle est marquée au niveau formel par l'accusatif.

Mots clés : relations spatiales, le mouvement, le cas, la préposition, la trajectoire, le français, le serbe.

\section{INTRODUCTION}

Dans l'étude des relations spatiales le mouvement représente une relation dynamique, plus complexe par rapport aux relations statiques. La complexité du mouvement provient du fait que c'est un phénomène spatiotemporel et il suppose le déplacement d'une cible dans l'espace qui se déroule dans le temps. C. Vandeloise (1987) souligne l'opposition entre une cible stable, facilement repérable et une cible mobile, dont la position mobile rend sa localisation plus difficile.

Le phénomène du mouvement a suscité beaucoup d'intérêt et d'analyses linguistiques surtout parmi les linguistes d'orientation sémantique cognitive (Talmy, 2000 ; Jackendoff 1991; 1996) à cause du lien entre les relations spatiales et les relations temporelles. Dans la littérature française beaucoup de linguistes se sont penchés sur le phénomène du mouvement (Gougenheim, 1959 ; Vandeoise, 1987 ; Boons, 1987 ; Borillo, 1998) et le rôle des prépositions (Vandeloise, 1986 ; 1988 ; Laur, 1993 ; Borillo, 1993 ; Ašić, 2008). De son côté, le serbe représente aussi un domaine intéressant pour l'étude du phénomène du mouvement vu que les relations spatiales y sont

\footnotetext{
*ivavilic@ff.uns.ac.rs
} 
exprimées par les cas, mais aussi par les prépositions. Les relations spatiales en serbe ont été étudiées par de nombreux linguistes, dont les plus remarquables sont M. Ivić et P. Piper (Ivić, 1957 ; 1957-1958 ; Piper, 2001 ; Piper et al., 2005). L'analyse contrastive entre les manières d'expression du mouvement en français et en serbe donne la possibilité d'envisager cette question sous un autre jour, surtout le rôle des prépositions dans les deux langues.

\section{LE MOUVEMENT ET LE DÉPLACEMENT}

Dans la littérature française traitant de l'expression des relations spatiales dynamiques, la question de différence entre le mouvement et le déplacement se pose souvent. D’après J.P. Boons les verbes de déplacement sont les verbes de mouvement qui expriment «le changement obligatoire du lieu d'un corps ne subissant par ailleurs aucune modification de forme ni de substance au cours du procès »(Boons, 1987 : 5). Le changement de lieu apparaît comme la notion clé dans la distinction entre le mouvement, qui peut être vu comme un changement de position ou de posture, et le déplacement, qui peut être considéré comme un phénomène de nature spatio-temporelle parce qu'il entraîne une relation complexe de modification des relations spatiales (Borillo, 1998 : 38). Pour C. Vandeloise le mouvement représente une notion plus générale, puisqu'il n'y a pas de déplacement sans mouvement (Vandeloise, 1987 : 84) Quelle que soit la définition de ces deux notions complexes, toutes les deux se rapportent au phénomène de structuration de l'espace, de conceptualisation des relations qui y sont présentes et qui sont exprimées par la langue.

Dans son analyse de conceptualisation de la structure spatiale, L. Talmy (Talmy, $2000: 180$ ) distingue deux sous-systèmes : le premier sous-système comprend les délinéations schématiques de n'importe quelle portion de l'espace et il peut être vu comme le cadre qui contient et localise les configurations et les interrelations du matériel (l'un ou plusieurs objets) du deuxième sous-système. Le premier sous-système inclut les concepts statiques (la région et la location) et les concepts dynamiques, dont les éléments essentiels sont la trajectoire et l'emplacement. Dans cette perspective, le 
mouvement et le déplacement représentent des phénomènes liés au changement de lieu ou de position d'un ou de plusieurs objets qui s'effectue dans le temps et dans l'espace, ce qui est indiqué par la trajectoire ${ }^{1}$.

Nous allons accepter la définition que le déplacement, en tant qu'une forme de mouvement, comprend le changement de lieu d'une cible, et nous allons considérer l'expression linguistique de ce phénomène en français et en serbe $^{2}$. Dans une telle perspective trois éléments s'avèrent fondamentaux : l'origine du déplacement, son terme et la trajectoire qui lie ces deux éléments.

\section{L'ORIGINE DU DÉPLACEMENT}

En français, l'origine du déplacement est marquée par le verbe qui, par sa signification intrinsèque, implique un lieu initial représentant le point à partir duquel le déplacement s'effectue (partir, sortir, s'éloigner). Le deuxième élément qui accompagne les verbes de ce type est la préposition. Pour marquer l'origine du déplacement le français utilise la préposition de (depuis): partir de la ville, sortir de la maison, s'éloigner du mur. C. Vandeloise souligne que de ne fait pas partie de prépositions statiques, à la différence des autres prépositions françaises après les verbes de mouvement (surtout la préposition à) et qu'elle implique un déplacement pratiquement toujours (Vandeloise, 1987 : 102) :

(1) Je voyais la frégate partant de Toulon dans la nuit... (AD/LM)

(1a) Video sam brod kako u noći kreće iz Tulona... (AD/PM)

(2) ... pendant que le fruit nous arrive directement $d u$ Midi à pleines caisses, l'arbre, ..., de la serre chaude où il passe l'hiver, ne fait qu'une courte apparition au plein air des jardins publics. (AD/LM)

(2a) ... jer nam voće dolazi direktno s juga u punim sanducima, a narandžino drvo,..., samo se kratko vrijeme pojavljuje iz svojih staklenih bašta u kojima provodi zimu, u našim javnim parkovima. (AD/PM)

1 Les verbes qui désignent le changement de position ou de posture comme s'agenouiller - kleknuti, se lever - ustati, se pencher - nagnuti se, etc. et qui possèdent le trait dynamique sans qu'il y ait effectivement de changement de lieu, indiquent aussi la trajectoire le long de laquelle le mouvement du corps ou d'une partie du corps se déroule.

${ }^{2}$ Dans notre analyse nous n'allons pas aborder les verbes qui expriment le changement de posture ou de position. 
L'exemple (1) illustre l'emploi du verbe partir et la préposition de pour marquer l'origine du déplacement. Dans l'exemple (2) la préposition de est placée après le verbe arriver qui ne marque pas le point de départ par sa signification intrinsèque (comme d'ailleurs ni le syntagme faire apparition), mais elle y indique tout de même le point initial de l'action verbale.

En serbe, comme en français, le verbe par sa signification intrinsèque exprime le point initial à partir duquel le déplacement se déroule. Cette signification fait partie du sens aspectuel et les verbes marquant l'origine du déplacement sont pour la plupart d'entre eux les verbes perfectifs (poći, izaći, udaljiti se). Deux autres éléments exprimant le point du départ sont le cas et la préposition. Pour marquer l'origine du déplacement le serbe utilise le génitif et les prépositions $i z, o d, s(a)$ comme dans les exemples (1a) et (2a): kreće iz Tulona, dolazi s juga, pojavljuje se iz staklene bašte. Et comme en français, même après un verbe dont la signification intrinsèque indique le terme du déplacement, le génitif et les prépositions $i z, o d, s(a)$ expriment l'origine du départ (dolaze s juga).

Le fait que dans les deux langues les prépositions $d e-i z$, od, $s(a)$ et en serbe aussi le génitif expriment l'origine du déplacement après un verbe comme arriver - stići dont la signification porte sur le terme du déplacement, indique le rôle prédominant de la préposition par rapport au verbe dans la localisation de la cible.

\section{LE TERME DU DÉPLACEMENT}

Le mouvement d'une cible mobile se déroule le long de la trajectoire vers un but qui peut correspondre au point final de la trajectoire ou peut simplement figurer comme le point visé sans être atteint. La différence entre le point final atteint ou visé lors du mouvement de la cible est généralement marquée dans la langue vu qu'elle relève du phénomène perceptif qui est la limite ou le bornage du chemin ${ }^{3}$. Le français et le serbe expriment précisément la distinction si le terme du déplacement est atteint ou non.

${ }^{3}$ Dans son explication de catégorie sémantique de télicité, R. Jackendoff (Jackendoff, 1996) part des verbes de mouvement pour expliquer l'importance du bornage de la trajectoire. À cause du lien étroit qui existe entre la trajectoire, vue comme une entité spatiale, et le temps qui passe lors du mouvement d'une cible mobile, le bornage de la 
En parlant de l'expression du mouvement d'approche en français, P. Charaudeau (1992 : 417-418) dit que ce mouvement est exprimé par un verbe de déplacement qui se combine avec l'une des prépositions : à, jusqu'à, vers, pour. Les prépositions à et jusqu'à marquent que le point final est atteint, alors que les prépositions vers et pour sont utilisées pour indiquer que le terminus n'est pas atteint.

Le rôle des prépositions après les verbes de mouvement par rapport au terme du déplacement a été étudié plus profondément par C. Vandeloise (1987) qui a souligné surtout la complexité de la préposition à. Pour lui, cette complexité provient d'abord du fait que la préposition à exprime aussi bien les relations statiques (il est au bureau - on je u kancelariji) que les relations dynamiques (il va au bureau - ide u kancelariju). L'analyse proposée par C. Vandeloise $(1987,1988)$ ouvre un champ de questions sur l'emploi de la préposition à et des autres prépositions statiques (sur, dans) en relation avec les verbes de déplacement, ce qui est illustré par les exemples (3)-(10a) :

(3) La mule était au bas de l'escalier, ... prête à partir pour la vigne... $(\mathrm{AD} / \mathrm{LM})$

(3a) Mazga je stajala udno stepenica, ... spremna da krene u vinograd... $(\mathrm{AD} / \mathrm{PM})$

(4) ... le premier moutardier salua d'un air galant et se dirigea vers le haut perron... (AD/LM)

(4a) ... prvi slačičar se otmeno pokloni, pa se uputi prema uzdignutom prilazu... (AD/PM)

(5) Il monte au grenier... (AD/LM)

(5a) On se pope na tavan ... (AD/PM)

(6) Par fournées et à pleine porte, on entre là, mes frères, comme le dimanche vous entrez au cabaret. (AD/LM)

(6a) Tu se ulazi u gomili kroz širom otvorena vrata, kao što vi nedjeljom ulazite u mehanu. (AD/PM)

(7) Quand la chèvre blanche arriva dans la montagne ... (AD/LM)

trajectoire affecte la télicité d'une situation verbale : quand la trajectoire est bornée, le temps est limité aussi et l'action verbale est vue comme télique; dans le cas de trajectoire sans point de limite, l'action verbale se déroule sans interruption dans le temps et la situation verbale est interprétée comme atélique. 
(7a) Kad bijela koza stiže u planinu ... (AD/PM)

(8) À ce moment les ânes arrivent sur la plate-forme... (AD/LM)

(8a) U tom trenutku stigoše magarci pred vjetrenjaču... (AD/PM)

(9) Puis il allait vers le fond en appelant : ... (AD/LM)

(9a) Zatim priđe izlazu, pa zovnu : ... (AD/PM)

(10) - Je veux aller dans la montagne, monsieur Seguin. (AD/LM)

(10a) - Hoću da odem u planinu, gospodine Segene. (AD/PM)

Dans les exemples (3) et (4) la cible se déplace (se dirigea vers) ou se prépare à se déplacer (prête à partir pour) à partir du point d'origine et ensuite le long de la trajectoire vers un but sans qu'on sache s'il sera atteint. L'idée du mouvement d'approche du but visé est marqué par les prépositions vers et pour après les verbes de mouvement se diriger et partir. Dans les exemples (5) et (6), par contre, le point final du déplacement indiqué par les verbes monter et entrer a été atteint, ce qui est marqué par la préposition à. Dans les exemples (7) et (8) après le verbe arriver ce sont les prépositions statiques dans et sur qui sont utilisées et dans les deux cas le point final a été atteint après le déplacement. L'exemple (9) illustre l'emploi de la préposition vers après le verbe aller qui peut se combiner avec cette préposition aussi bien qu'avec la préposition à.

Comment expliquer l'emploi de ces prépositions ? Considérés du point de vue du terme du déplacement, en relation avec la trajectoire qui mène vers ce terme, les verbes de mouvement se distinguent par rapport au type de déplacement qu'ils expriment. La signification intrinsèque de certains verbes, comme arriver ou entrer, comprend le point final du déplacement, alors que certains verbes comme aller expriment le mouvement de la cible le long de la trajectoire d'une manière générale. Similairement, le verbe monter (ou descendre) précise seulement que le mouvement est orienté par rapport à l'axe vertical. Certains verbes, comme partir ou se diriger, indiquent le point initial du départ à partir duquel le déplacement de la cible reste imprécis. Vu que la signification des verbes mêmes porte sur les différentes étapes du mouvement le long de la trajectoire, l'emploi des prépositions dépend aussi de la perspective de localisation de la cible. Ce fait a mené $\mathrm{C}$. Vandeloise à formuler le principe d'anticipation (Vandeloise 1987) et à faire la distinction entre les verbes qui expriment la localisation effective de la cible, comme arriver, entrer, marcher, et les verbes qui ne le font pas, comme aller, partir, monter, descendre et après lesquels les prépositions statiques décrivent la position anticipée de la 
cible après le mouvement. C'est par le principe d'anticipation que l'emploi des prépositions à et dans peut être expliqué dans les exemples (5) et (10) avec les verbes monter et aller dont la signification n'exprime pas la localisation effective de la cible : après le mouvement elle est au grenier (5) et dans la montagne (10). Après le verbe arriver (les exemples (7) et (8)) les prépositions dans et sur indiquent aussi la position de la cible après le mouvement. Ces prépositions apportent aussi les informations sur les propriétés dimensionnelles du site et les relations contenant / contenu et porteur / porté, à la différence de la préposition à qui pourrait aussi être utilisée après ce verbe, mais qui présenterait le site comme un point sur la trajectoire. C'est le cas avec le verbe entrer dans l'exemple (6) où la préposition à marque le point final sur la trajectoire, alors que ce verbe peut se combiner aussi avec la préposition dans qui serait utilisé dans l'expression d'une relation statique (il est dans le cabaret) ou dynamique (il entre dans la chambre). Dans le cas des verbes qui expriment l'origine du départ (les exemples (3) et (4)), les prépositions vers et pour indiquent le déplacement le long de la trajectoire sans qu'on sache si le but visé sera atteint ou non.

D. Laur (Laur, 1993) parle aussi de l'interrelation qui existe entre les verbes de déplacement et les prépositions en français. Cet auteur distingue les verbes qui indiquent le lieu initial, final ou médian par rapport auquel s'effectue le déplacement, d'un côté, et les prépositions qui possèdent aussi les mêmes traits initial, final ou médian, de l'autre côté. La combinatoire des traits sémantiques de ces deux classes de mots ne se fait pas de manière aléatoire, mais suit une certaine logique. C'est ainsi qu'un verbe exprimant le trait final (arriver) ne peut pas se combiner avec une préposition directionnelle qui indique que le terme n'a pas été atteint, comme vers (*arriver vers). La combinaison d'un verbe initial (partir) avec une préposition finale comme jusqu'à n'est pas permise non plus. Dans cette combinatoire, les deux classes apportent leur signification :

quelle que soit la combinaison concernée, le verbe détermine toujours la propriété 'changement de lieu ou orientation', cette propriété lui étant spécifique... La préposition détermine quant à elle la localisation interne ou externe de la cible par rapport au lieu de référence à l'issue du procès (Laur, 1993 : 65).

Les traductions des exemples cités dessus dévoilent les ressemblances (l'expression de la direction vers le point terminal, comme dans les exemples (3-3a), (4-4a) et (9-9a)) et les différences (l'emploi des prépositions $n a$, $u$ dans 
les exemples (5-5a) et (6-6a)) entre le français et le serbe. La plus grande différence se rapporte au fait que le serbe utilise aussi le système casuel pour exprimer si le but a été atteint ou non.

En serbe, le terme du déplacement est marqué par les cas, le datif, l'accusatif et le génitif, précédés de prépositions de lieu $\mathrm{ka}$, prema/u, na/do. Le système complexe des cas spatiaux et leur signification par rapport au mouvement d'approche de but a été expliqué en détail (Ivić, 1957, 1957-1958 ; Piper et al., 2005). M. Ivić définit le datif et l'accusatif comme les cas dynamiques qui indiquent le but de l'action verbale, l'accusatif exprimant le terminus de l'action et le datif marquant la direction de l'action sans préciser si le point final est effectivement atteint ou non (Ivić, 1957). Le génitif, en général, a la caractéristique d'exprimer le rapport précis entre deux entités et dans le sens spatial il marque les limites, initiale - od et finale - do (Ivić, 19571958). Les exemples (11)-(13) illustrent l'emploi des cas pour marquer le mouvement vers le point final en serbe :

(11) Tada i najsnažniji kasabalijski bekrija leti kao lutka od krpâ i slame $k a$ vratima, koja je Malčika već otvorila u pravom trenutku, pa kroz vrata pravo na ulicu. (IA/DĆ)

(11a) Le plus costaud des noceurs volait alors comme une poupée de chiffon par la porte, ouverte par Maltchika à l'instant propice, et se retrouvait dans la rue. (IA/PD)

(12) Nju su posle odveli u konak, ... (IA/DĆ)

(12a) Quant à elle, on l'avait emmené au palais, ... (IA/PD)

(13) Stigli su do kapije. (IA/DĆ)

(13a) Ils parvinrent à la kapia. (IA/PD)

Dans l'exemple (11) le datif ( $k a$ vratima) indique la direction vers le but, alors que l'accusatif (na ulicu) exprime que la cible a effectivement atteint le terminus. Le site de l'exemple (12) (u konak) est également exprimé par l'accusatif, précédé de préposition $u$ qui indique, comme la préposition $n a$ de l'exemple (11), les caractéristiques dimensionnelles du site ${ }^{4}$. Le génitif dans l'exemple (13) (do kapije) indique la limite finale du mouvement.

\footnotetext{
${ }^{4}$ Notons aussi que les prépositions $u$, na sont utilisées avec le locatif pour exprimer la relation statique.
} 
À part les cas, les verbes et leurs caractéristiques aspectuelles ont aussi un rôle important dans l'expression du mouvement d'approche de but. Les traits aspectuels des verbes sont liés à la trajectoire et à la façon de laquelle le mouvement se déroule. Quand ce mouvement est continu en direction du but visé, c'est un verbe imperfectif qui est utilisé pour exprimer l'action continue, comme dans l'exemple (11) - leti ka vratima. Si le point final est atteint lors du mouvement, c'est un verbe perfectif qui est employé, comme dans les exemples (12) et (13) - odveli u konak, stigli su do kapije. Dans l'exemple (11) il manque le verbe pour indiquer le mouvement jusqu'au site à l'accusatif (na ulicu), mais ce serait un verbe perfectif (izleteti, pasti) qui aurait été utilisé.

En serbe aussi, on remarque certaines contraintes sur les possibilités de combinatoire entre les verbes de déplacement et les cas précédés de prépositions de lieu. Ces contraintes sont liées aux traits aspectuels du verbe, mais aussi à la signification intrinsèque du verbe par rapport au terme du déplacement. Un verbe perfectif qui indique que le terminus est atteint ne peut pas se combiner avec le datif qui marque le mouvement en direction du terme ( ${ }^{*}$ stići ka, prema). Un verbe imperfectif ne serait pas utilisé pour exprimer que le point final est atteint ( $*$ leteti $u, n a+N_{A c c}$ ), mais il peut marquer l'arrivée à la limite du chemin (leteti do $+N_{\text {Gén }}$ ).

\section{LA TRAJECTOIRE}

L'origine et le terme du déplacement sont liés par la trajectoire qui représente l'élément essentiel dans le mouvement d'une cible mobile. C'est la trajectoire qui véhicule le trait de dynamisme, par opposition à l'état, statique. Dans l'analyse de l'expression de trajectoire en français, nous citons deux linguistes, G. Gougenheim (1959) et C. Vandeloise (1988) qui soulignent l'importance de la préposition à pour l'expression des relations dynamiques aussi bien que des relations statiques.

Pour G. Gougenheim (1959) la valeur essentielle de la préposition à réside dans son caractère ponctuel, «le lieu où l'on va et le lieu où l'on se trouve étant exprimés de la même façon par la fusion de ces catégories » (Gougenheim, 1959 : 8). Même après un verbe de mouvement la préposition à garde son caractère de préposition statique, et c'est uniquement le verbe qui véhicule le trait dynamique. Il souligne que déjà l'origine de cette préposition, la préposition latine $a d$ (suivie d'un nom à l'accusatif) avait un sens local, la direction du mouvement, et un sens final, qui se sont maintenus en français 
moderne. La propriété de représenter le lieu comme un point dans l'espace distingue pourtant la préposition à des prépositions sur et dans qui présentent le lieu comme surface ou comme volume et c'est par cette propriété que à en français participe à l'indication de la direction du mouvement.

Pour Claude Vandeloise $(1987,1988)$ la préposition à a un caractère localisateur et la fonction de permettre au destinataire du message de s'orienter par rapport au site et à la cible. En parlant de son caractère ponctuel, il dit que les objets introduits par la préposition à sont conceptualisés ponctuellement quelle que soit leur dimensionnalité physique réelle parce qu'ils ont la fonction de repère. C'est l'idée de recherche qui se profile souvent derrière la localisation par la préposition à. Comme elle maximalise l'asymétrie entre la cible et le site, le site introduit par la préposition à est souvent éloigné du locuteur. La distance donne au site une allure ponctuelle, ce qui indique la direction et le chemin de recherche de la cible.

Pour illustrer l'usage de la préposition à pour indiquer le chemin de recherche, C. Vandeloise donne l'exemple suivant : «Allo, Léopold, je suis à l'arène» (Vandeloise, 1988 : 132). Même si le locuteur se trouve effectivement à l'intérieur du bâtiment, il choisira la préposition à pour faciliter la recherche de son interlocuteur. La préposition à marquerait ainsi, d'une manière implicite, la trajectoire.

En serbe, par contre, la trajectoire est marquée au niveau formel par l'accusatif qui exprime la relation dynamique par opposition au locatif qui exprime la relation statique ${ }^{5}$. En parlant de la valeur de l'accusatif dans le système casuel serbe, M. Ivić souligne la valeur dynamique comme le trait principal de ce cas. À part de marquer le terminus de l'action verbale (avec les prépositions $u, n a, o$ ), l'accusatif marque aussi la totalité de l'action verbale avec les prépositions $u z, n i z$, kroz (Ivić, 1957-1958 : 151). Nous ajoutons que les prépositions $u z$, niz, kroz désignent l'espace unidimensionnel ce qui peut

${ }^{5}$ Le locatif en serbe exprime le lieu de l'action verbale, qui peut comprendre le trait de dynamisme (trči po parku-il court dans le park) (Ivić, 1957 : 151). Ce lieu, pourtant, en tant que la région qui contient l'action verbale, fait partie du concept statique dans la structuration de l'espace (Talmy 2000). 
traduire aussi sa caractéristique de marquer la trajectoire ${ }^{6}$. Les exemples (1416a) illustrent l'emploi de l'accusatif en serbe :

(14) Ti ne dođe večeras $u$ han? (IA/DĆ)

(14a) Tu n'es pas venu à l'auberge ce soir? (IA/PD)

(15) Kad su došli na pijac, iz opreza nisu krenuli pravo kroz čaršiju,... (IA/DĆ)

(15a) Arrivés au marché, par prudence, ils ne traversèrent pas le bazar,... (IA/PD)

(16) Iznad mosta, nepun sat hoda $u z$ vodu, ... (IA/DĆ)

(16a) En amont du pont, à moins d'une heure de marche, ... (IA/PD)

Après les verbes de mouvement doći, stići dans les exemples (14-15a), en serbe, c'est l'accusatif ( $u$ han, na pijac) qui marque le terme du déplacement, et par conséquent, la trajectoire. Dans la traduction en français, c'est la préposition à (à l'auberge, au marché) qui est utilisée. Les prépositions serbes kroz, uz (les exemples (15-16a)) désignent le déplacement de la cible le long de la trajectoire qui pourrait être représentée comme une ligne droite (kroz čaršiju, uz vodu). La traduction française exprime la même signification, par le verbe traverser et la locution adverbiale en amont.

\section{CONCLUSION}

L'analyse de l'expression du mouvement en français et en serbe montre que les deux langues marquent les éléments essentiels du déplacement : l'origine du départ, son terme et la trajectoire. Dans les deux langues, le verbe, par sa signification intrinsèque de marquer les points initial ou final, joue un rôle important, mais la préposition en français et la construction préposition + cas en serbe y ont aussi un impact important.

Dans l'expression de l'origine du départ, en français, à part le verbe, c'est la préposition de, essentiellement dynamique, qui marque le déroulement de l'action depuis le point initial, alors qu'en serbe, ce sont les prépositions od,

${ }^{6}$ Dans l'élaboration des catégories conceptuelles, R. Jackendoff (1991) définit la dimensionnalité comme la catégorie à quatre valeurs : le point est 0 - dim, la ligne est 1 - dim, la surface est 2 - dim et le volume est 3 - dim. La trajectoire, l'une des catégories conceptuelles fondamentales, est conceptualisée comme la ligne, 1 - dimensionnelle. 
$i z, s(a)$ et le génitif. Dans les deux langues, ces prépositions et, en serbe, le génitif, indiquent l'origine du départ même après un verbe désignant par sa signification propre le point final (comme arriver).

En ce qui concerne le terme du déplacement, les deux langues distinguent si le point final est atteint ou non. Pour exprimer que le terme est visé, mais pas atteint, le français utilise les prépositions vers et pour, et le serbe se sert des prépositions $k a$, prema et du datif. L'expression du point final atteint s'avère plus complexe en français qui utilise les prépositions statiques $\grave{a}$, sur, dans, qui, avec le verbe, marquent souvent la position de la cible après le mouvement. En serbe, ce sont aussi les prépositions statiques $u$, na et l'accusatif qui indiquent le point final atteint.

La plus grande différence entre le français et le serbe s'observe dans l'expression de la trajectoire. En français, la trajectoire est exprimée de manière implicite par le verbe et la préposition $\grave{a}$, alors qu'en serbe elle est marquée au niveau formel par l'accusatif précédé de prépositions $u, n a, u z, n i z$, kroz.

Ivana Vilić

MOVEMENT IN THE EXPRESSION OF SPATIAL RELATIONS IN FRENCH

\section{AND IN SERBIAN}

\section{Summary}

The paper presents the analysis of the expression of movement in French and in Serbian from the perspective of three basic elements, the origin of movement, its term and its path. In both languages the verb plays an important part along with the prepositions in French and the preposition + case in Serbian. The origin in French is expressed by the preposition de, essentially dynamic, while in Serbian it is marked by genitive preceded by prepositions $o d, i z, s(a)$. Both languages make distinction if the term is reached or not. The movement toward the final point in French is expressed by the prepositions vers, pour, and in Serbian by dative and prepositions ka, prema. Attained final point in French is marked by the static prepositions $\grave{a}$, sur, dans which often express the position of the object after the motion. In Serbian the accusative with the prepositions $u, n a$ indicates that the term is reached. The most salient difference between the French and the Serbian is the expression of the path: in French it is expressed by the verb and the preposition $\grave{a}$, while in Serbian it is marked on the formal level by the accusative. 
Key words: spatial relations; movement; case; preposition; path; French; Serbian.

\section{RÉFÉRENCES BIBLIOGRAPHIQUES}

Ašić. T. (2008). Espace, Temps, Prépositions. Genève : Librairie Droz S.A.

Boons, J.P. (1987). La notion sémantique de déplacement dans une classification syntaxique des verbes locatifs. Langue Française, 76, 540.

Borillo, A. (1998). L'espace et son expression en français. Paris : Ophrys.

Charaudeau, P. (1992). Grammaire du Sens et de l'Expression. Paris : Hachette.

Gougenheim, G. (1959). Y a-t-il des prépositions vides en français? Le Français moderne, 27, 1-25.

Ivić, M. (1957). Jedno poglavlje iz gramatike našeg modernog jezika - sistem mesnih padeža. Godišnjak Filozofskog fakulteta u Novom Sadu, 2, 145158.

Ivić, M. (1957-1958). Sistem predloških konstrukcija u srpskohrvatskom jeziku. Južnoslovenski filolog, vol. 22, n 1-4, 141-161.

Jackendoff, R. (1991). Parts and boundaries. Cognition, 41, 9-45.

Jackendoff, R. (1996). The Proper Treatment of Measuring Out, Telicity, and Perhaps Even Quantification in English. Natural Language \& Linguistic Theory, vol. 14, $\mathrm{n}^{\circ} 2,305-354$.

Laur, D. (1993). La relation entre le verbe et la préposition dans la sémantique du déplacement. Languages, 110, 47-67.

Piper, P. (2001). Jezik i prostor. Beograd : Čigoja štampa.

Piper, P. et al. (2005). Sintaksa savremenoga srpskog jezika. Beograd-Novi Sad: Institut za srpski jezik SANU-Beogradska knjiga-Matica srpska, 2005.

Talmy, L. (2000). Toward a Cognitive Semantics I. Cambridge : MIT Press.

Vandeloise, C. (1986). L'espace en français. Paris : Éditions du Seuil.

Vandeloise, C. (1987). La préposition à et le principe d'anticipation. Langue Française, 76, 77-111.

Vandeloise, C. (1988). Les usages spatiaux statiques de la préposition à. Cahiers de lexicologie, 53, 119-148.

\section{CORPUS}

AD/LM - Daudet, A. (1985). Lettres de mon moulin. Paris : Le Livre de Poche. 
AD/PM - Dode, A. (1963). Pisma iz mog mlina. Sarajevo : Veselin Masleša. (traduction: Tanja Dugonjić).

IA/DĆ - Andrić, I. (1976). Na Drini ćuprija. Beograd : Prosveta.

IA/PD - Andrić, I. (1994). Le Pont sur la Drina. Paris : Belfond. (traduction: Pascale Delpech). 